\title{
Economic benefit of carpal tunnel release in the Medicare patient population
}

\author{
Zachary S. Hubbard, BS, ${ }^{1}$ Tsun Yee Law, MD, MBA, ${ }^{2}$ Samuel Rosas, MD, ${ }^{3}$ \\ Sarah C. Jernigan, MD, MPH, ${ }^{4}$ and Harvey Chim, MD ${ }^{5}$ \\ 1 University of Miami Miller School of Medicine, Miami, Florida; ${ }^{2} \mathrm{Holy}$ Cross Orthopedic Institute, Fort Lauderdale, Florida; \\ ${ }^{3}$ Department of Orthopaedics, Wake Forest School of Medicine, Winston-Salem, North Carolina; ${ }^{4}$ Department of Neurosurgery, \\ Carolina Neurosurgery and Spine Associates, Charlotte, North Carolina; 5Division of Plastic and Reconstructive Surgery, \\ University of Florida College of Medicine, Gainesville, Florida
}

OBJECTIVE The epidemiology of carpal tunnel syndrome (CTS) has been extensively researched. However, data describing the economic burden of CTS is limited. The purpose of this study was to quantify the disease burden of CTS and determine the economic benefit of its surgical management.

METHODS The authors utilized the PearlDiver database to identify the number of individuals with CTS in the Medicare patient population, and then utilized CPT codes to identify which individuals underwent surgical management. These data were used to calculate the total number of disability-adjusted life years (DALYs) associated with CTS. A human capital approach was employed and gross national income per capita was used to calculate the economic burden.

RESULTS From 2005 to 2012 there were 1,500,603 individuals identified in the Medicare patient population with the diagnosis of CTS. Without conservative or surgical management, this results in 804,113 DALYs without age weighting and discounting, and 450,235 DALYs with age weighting and a discount rate of 3\%. This amounts to between $\$ 21.8$ and $\$ 39$ billion in total economic burden, or $\$ 2.7-\$ 4.8$ billion per year. Surgical management of CTS has resulted in the aversion of 173,000-309,000 DALYs. This has yielded between $\$ 780$ million and $\$ 1.6$ billion in economic benefit per year. Endoscopic carpal tunnel release provided between $\$ 11,683$ and $\$ 23,186$ per patient at $100 \%$ success while open carpal tunnel release provided between $\$ 10,711$ and $\$ 22,132$ per patient at $100 \%$ success. The benefit-cost ratio at its most conservative is $2.7: 1$, yet could be as high as $6.9: 1$.

CONCLUSIONS CTS is prevalent in the Medicare patient population, and is associated with a large amount of economic burden. The surgical management of CTS leads to a large reduction in this burden, yielding extraordinary economic benefit.

https://thejns.org/doi/abs/10.3171/2018.1.FOCUS17802

KEYWORDS carpal tunnel release; DALY; economic benefit; utilization; disability-adjusted life year

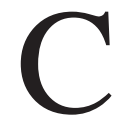
ARPAL tunnel syndrome (CTS) is the most commonly diagnosed peripheral neuropathy in the US, with an incidence of approximately 1-3 people per 1000 per year. ${ }^{3,6,22}$ Conservative therapy is typically used, but many patients ultimately require surgery. This is one of the most common surgical procedures performed each year with more than 600,000 cases performed annually. ${ }^{22}$
Given the prominence of this disease, a study determining disease burden and economic benefit of treating the disease is of value. The onset of disease for CTS is reportedly between 40 and 60 years of age, therefore the Medicare patient population would be greatly impacted by this condition. ${ }^{9,21}$ The authors are unaware of any prior economic analysis performed within the Medicare patient popula-

ABBREVIATIONS CPT = current procedural terminology; $C T S=$ carpal tunnel syndrome; DALY = disability-adjusted life year; ECTR = endoscopic carpal tunnel release; $\mathrm{GNI}=$ gross national income; OCTR = open carpal tunnel release; USD = US dollars.

SUBMITTED January 2, 2018. ACCEPTED January 31, 2018.

INCLUDE WHEN CITING DOI: 10.3171/2018.1.FOCUS17802. 
tion. The prevalence of CTS in the Medicare patient population leads to a large amount of disease burden that can be averted by surgical management. There is an increased focus on cost effectiveness and cost containment in medicine, and as such an understanding of health care resource utilization and economic burden for CTS would be valuable. The purpose of this study was to describe the disease burden of CTS in the Medicare patient population and to evaluate the benefit of providing surgery from a disease burden aversion model.

\section{Methods \\ Data Collection}

To assess the economic benefit of surgery for CTS, a previously reported utilization study was performed by the authors..$^{13}$ A retrospective review of a Medicare database within the PearlDiver supercomputer was performed for patients undergoing open carpal tunnel release (OCTR) or endoscopic carpal tunnel release (ECTR) from 2005 to 2012. The PearlDiver database is a publicly available Health Insurance Portability and Accountability Actcompliant national database compiled from a collection of private payer records. This database contains current procedural terminology (CPT) and International Classification of Diseases, Ninth Revision (ICD-9) codes. Patients who underwent OCTR were identified by CPT code 64721 and ICD-9 code 04.43. ECTR was identified by CPT code 29848. CTS was identified by ICD-9 code 354.0.

\section{Disability-Adjusted Life Years}

The disability-adjusted life year (DALY) was created in the early 1990s as a health measure that extended beyond deaths avoided. It encompasses healthy years of life lost as a result of disability as well as years of life lost due to premature death. ${ }^{19}$ The DALY itself is calculated by assigning a particular health state with a corresponding disability weight ranging from 0 to $1(0=$ perfect health, $1=$ death). The value of a DALY is impacted based on adjustments made to discounting and age weighting. Discounting arises from the belief that individuals value a healthy year of life at the present, decreasing the value of a life year in the future. Age weighting places more value on a year of middle life compared to those at the beginning or end of life. Due to the debate over the accuracy of these principles, calculations were made with and without age weighting and discounting. ${ }^{20}$

\section{Calculating DALYs}

DALYs were calculated and converted to US dollars by means of the human capital approach. The human capital approach assumes that the individual is valued at their contribution to the economy. The notation DALY $(\mathrm{r}, \mathrm{K}, \mathrm{C})$ represents the adjustments to the DALY based on discount-weighting, age weighting, and the age-weighting factor, respectively. For the human capital approach, DALYs $(3,1,0.04)$ were calculated under the conditions of $3 \%$ discounting + age weighting. Additionally, DALYs $(0,0,0)$ were calculated under the conditions without discounting or age weighting. DALYs were calculated using the following equation used in previous studies: ${ }^{5,20,26}$

$$
\begin{gathered}
D A L Y s=\int_{a}^{L}\left\{\left[K \cdot D W \cdot C x e^{-d x} e^{-r(x-a)}\right]\right. \\
\left.+\quad\left[D W \cdot(1-K) e^{-r(x-a)}\right]\right\} d x
\end{gathered}
$$

in which " $L$ " is the country-specific life expectancy in the US (www.worldbank.org), "a" represents the age of onset of disease, and "DW" represents the disability weight for

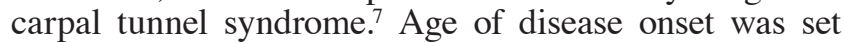
at 65 years of age to avoid overestimating the disability within the Medicare patient population. " $\mathrm{K}$ " is the ageweighting modulation constant $(0=$ no age weighting, 1 = age weighting), " $\mathrm{C}$ " is the age-weighting disability constant (0.04), " $\mathrm{x}$ " is the age integrated over the duration of disease and/or years of life lost, and " $r$ " is the discount rate $(3 \%){ }^{14}$

\section{Valuing DALYs}

The human capital approach values averted DALYs at a lower bound of DALYs $(3,1,0.04) \times$ gross national income (GNI) per capita and an upper bound of DALYs $(0,0,0)$ $\times$ GNI per capita. The GNI per capita was calculated by averaging the US GNI per capita reported by the World Bank (data.worldbank.org) from the years included in this study. ${ }^{26}$

\section{Determining Economic Benefit}

The DALY calculations allow one to interpret and quantify multiple health states. Importantly, this allows for the calculations of DALYs averted due to an intervention by establishing two scenarios that reflect the course of disease with and without intervention. In the first scenario, the assumption is made that no surgical intervention is performed, and DALYs are calculated. In the second scenario, DALYs are calculated when surgical intervention is conducted. The difference obtained by subtracting total DALYs in scenario 2 from scenario 1 is representative of the DALYs averted due to the intervention, and is representative of the improved health care state as a consequence. Economic benefit is the value of this difference in US dollars (USD). ${ }^{26}$ The assumption is that surgery for CTS relieves the disability weight associated with the condition. Calculations for economic benefit were made for $100 \%, 90 \%, 80 \%$, and $70 \%$ success rate, representing the proportion of DALYs averted by means of surgery.

\section{Results}

\section{Medicare Utilization}

Our query returned $1,500,603$ patients with CTS of which 507,924 (33.8\%) and 68,768 (4.6\%) were managed surgically with OCTR and ECTR, respectively (Fig. 1A). Surgeries for CTS were performed most frequently in the $<65$ age group. Both procedures were performed most commonly in females (64\%) compared with males (36\%; Fig. 1C). OCTR and ECTR were performed the most in the Southern region $(36.1 \%$ and $37.6 \%)$ and the least in the Western region (12.9\% and 14.7\%; Fig. 1B). Mean annual charges were significantly higher in the OCTR group 
CTS by age in the Medicare population
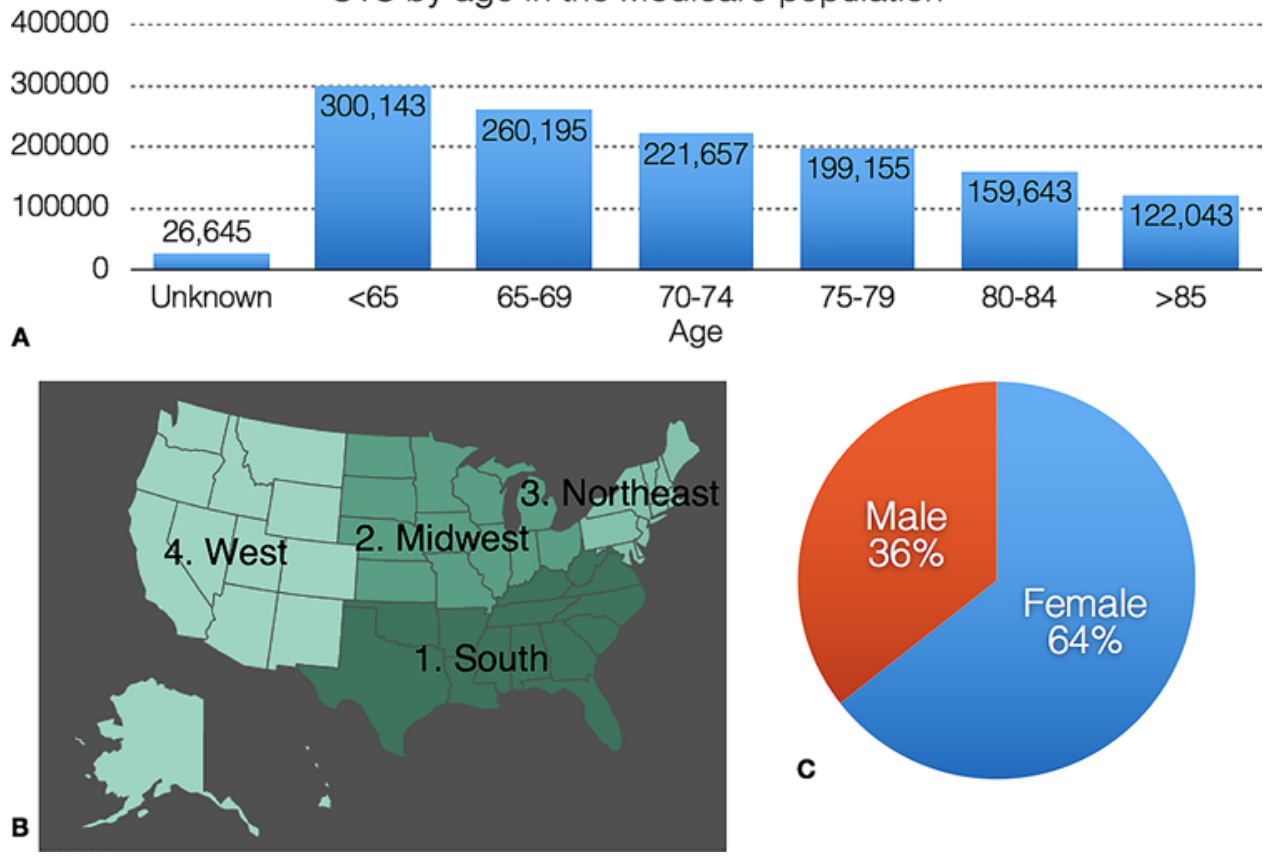

FIG. 1. A: Bar graph demonstrating the number of patients with CTS according to age group. B: Geographic representation of total CTS surgeries following US Census Bureau data. C: Pie chart demonstrating total CTS surgeries by sex.

(\$3820) than ECTR (\$2952). Conversely, reimbursements paid by Medicare were higher in ECTR (\$1643) than OCTR (\$1312; Fig. 2).

\section{DALYs}

DALYs were calculated for all 1,500,603 patients with CTS within the Medicare patient population. This resulted in a total of 450,235 DALYs calculated using age weighting and discounting, and a total of 804,113 DALYs cal-
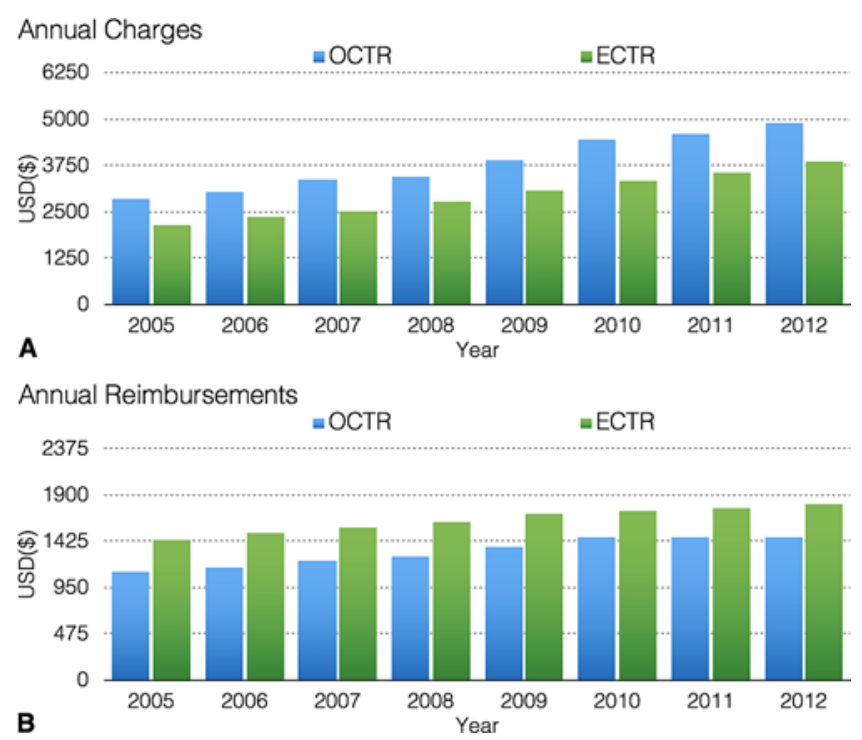

FIG. 2. Bar graphs demonstrating annual charges (A) and annual reimbursements (B) for OCTR and ECTR from 2005 to 2012. culated without age weighting and discounting (Table 1). CTS results in an average loss of 78,375 DALYs per year. Converting DALYs into USD, the total disease burden of carpal tunnel syndrome ranged from \$2.7 to \$4.8 billion annually or between $\$ 21.8$ and $\$ 39$ billion during the study period (Table 2 ).

\section{Economic Benefit}

In scenario 1 , a total of $1,500,603$ patients with CTS experienced a health state equivalent to $450,000-804,000$ total DALYs. In scenario 2, 576,692 of these patients underwent surgical management that resulted in an improved health care state of 277,000-495,000 total DALYs. Subtracting scenario 2 from scenario 1 resulted in a total of 173,000-309,000 total DALYs averted due to both OCTR and ECTR.

Converting averted DALYs to USD amounts to $\$ 8.39$ $\$ 14.98$ billion in total gross economic benefit for both OCTR and ECTR assuming a 100\% success rate, \$7.54$\$ 13.48$ billion assuming a 90\% success rate, $\$ 6.71-\$ 11.98$ billion assuming an $80 \%$ success rate, and $\$ 5.87-\$ 10.48$ assuming a $70 \%$ success rate. OCTR was responsible for

TABLE 1. DALYs calculated using a human capital approach

\begin{tabular}{ccc}
\hline DALY & $\begin{array}{c}3 \% \text { Discounting } \\
\operatorname{DALY}(3, \mathrm{~K}, \mathrm{C})\end{array}$ & $\begin{array}{c}\text { No Discounting } \\
\operatorname{DALY}(0, \mathrm{~K}, \mathrm{C})\end{array}$ \\
\hline Age weighting $(\mathrm{r}, 1,0.04)$ & 450,235 & 544,703 \\
\hline No age weighting $(r, 0,0)$ & 661,313 & 804,113 \\
\hline
\end{tabular}

$(r, K, C)=($ discount rate, age weighting modulation constant, age weighting disability constant) 
TABLE 2. Disease burden of CTS in the Medicare patient population

\begin{tabular}{lll}
\hline \multicolumn{1}{c}{ Variable } & DALY $(3,1,0.04)$ & DALY $(0,0,0)$ \\
\hline DALYs & 450,235 & 804,113 \\
\hline Annual economic burden & $\$ 2.7$ billion & $\$ 4.8$ billion \\
\hline Total economic burden & $\$ 21.8$ billion & $\$ 39$ billion \\
\hline
\end{tabular}

$(\mathrm{r}, \mathrm{K}, \mathrm{C})=($ discount rate, age weighting modulation constant, age weighting disability constant)

the greatest amount of gross benefit due to a greater number of procedures performed compared to ECTR.

OCTR and ECTR costs were generated from the average charges billed to Medicare at $\$ 3820$ and $\$ 2952$, respectively. The product of the average cost and the total number of procedures resulted in total costs of $\$ 1.94$ billion and \$203 million for OCTR and ECTR, respectively (Tables 3 and 4). Average annual costs amounted to \$242.5 million/year and \$25.4 million/year for OCTR and ECTR, respectively. These costs were used to determine the cost-benefit ratio for surgical intervention for CTS.

Subtracting total costs from total gross economic benefit yields the net economic benefit of surgeries for procedures performed within the Medicare patient population. Net economic benefit totaled between $\$ 6.2$ and $\$ 12.8$ billion, or an average between $\$ 780$ million and $\$ 1.60$ billion per year. Total net economic benefit for OCTR ranged between \$5.4 and \$11.2 billion (Table 3), while for ECTR it ranged between $\$ 803$ million and $\$ 1.59$ billion (Table 4). ECTR was associated with lower costs, therefore the net economic benefit was greater per patient. ECTR provided between $\$ 11,683$ and $\$ 23,186$ per patient at $100 \%$ success while OCTR provided between $\$ 10,711$ and $\$ 22,132$ per patient at $100 \%$ success. The most conservative estimate for CTS surgery was at a surgical success rate of $70 \%$. This success rate equaled $\$ 7498$ to $\$ 15,492$ per patient for OCTR and $\$ 8178$ to $\$ 16,230$ per patient for ECTR (Tables 3 and 4). The benefit-cost ratio at its most conservative is

TABLE 3. Net economic benefit of OCTR

\begin{tabular}{|c|c|c|}
\hline Variable & $\operatorname{DALY}(3,1,0.04)$ & $\operatorname{DALY}(0,0,0)$ \\
\hline Gross economic benefit & $8,386,905,542$ & $14,978,888,306$ \\
\hline OCTR economic benefit & $7,380,476,877$ & $13,181,421,709$ \\
\hline Cost per OCTR* & \multicolumn{2}{|c|}{3820} \\
\hline Total cost OCTR* & \multicolumn{2}{|c|}{$1,940,000,000$} \\
\hline Net economic benefit of OCTR & $5,440,476,877$ & $11,241,421,709$ \\
\hline \multicolumn{3}{|l|}{ Net economic benefit per patient $†$} \\
\hline $100 \%$ & 10,711 & 22,132 \\
\hline $90 \%$ & 9640 & 19,919 \\
\hline $80 \%$ & 8569 & 17,706 \\
\hline $70 \%$ & 7498 & 15,492 \\
\hline
\end{tabular}

TABLE 4. Net economic benefit of ECTR

\begin{tabular}{|c|c|c|}
\hline Variable & $\operatorname{DALY}(3,1,0.04)$ & $\operatorname{DALY}(0,0,0)$ \\
\hline Gross economic benefit & $8,386,905,542$ & $14,978,888,306$ \\
\hline ECTR economic benefit & $1,006,428,665$ & $1,797,466,597$ \\
\hline Cost per ECTR* & \multicolumn{2}{|c|}{2952} \\
\hline Total cost ECTR* & \multicolumn{2}{|c|}{$203,000,000$} \\
\hline Net economic benefit of ECTR & $803,428,665$ & $1,594,466,597$ \\
\hline \multicolumn{3}{|l|}{ Net economic benefit per patient $†$} \\
\hline $100 \%$ & 11,683 & 23,186 \\
\hline $90 \%$ & 10,515 & 20,868 \\
\hline $80 \%$ & 9347 & 18,549 \\
\hline $70 \%$ & 8178 & 16,230 \\
\hline
\end{tabular}

2.7:1, yet could be as high as 6.9:1 with greater surgical success.

\section{Discussion}

This study is the first to analyze the utilization, cost of treatment, disease burden, and economic benefit of treating CTS in the Medicare patient population. CTS is the most common peripheral neuropathy in the US and no treatment leads to mild to moderate disability. This study attempted to quantify that disability and the long-term benefit of surgical intervention.

CTS management has evolved over time with trends shifting from an open toward an endoscopic approach..$^{22}$ Although many studies have reported similar outcomes, the endoscopic approach is being utilized at a greater rate over the past two decades. ${ }^{8,10,17}$ This growth might be influenced by patient preference due to a reduction in scar tenderness and increase in grip and pinch strength. ${ }^{17,23} \mathrm{In}$ terestingly, financial factors might be present as well. Our study found that ECTR charges were lower, which is corroborated by the study of Trumble et al. who found that the average cost of OCTR was $\$ 3940$ compared with $\$ 3750$ for ECTR. ${ }^{24}$ These financial factors may impact patient and physician preference, but they also impact the net economic benefit provided to the population affected by CTS . Our study found higher net economic benefit provided by ECTR compared with OCTR, due to the lower charges.

Defining the surgical success rate can be difficult due to variations in both surgeon and patient factors across the Medicare population. For this reason, benefit was calculated at varying ranges of surgical success ranging from $70 \%$ to $100 \%$ success. Importantly, factors such as complication rate, reoperation rate, and patient satisfaction should be considered. Atroshi et al. reported the outcomes of 128 patients, 12.8 years following a randomized controlled trial for open versus endoscopic carpal tunnel release. There was similar efficacy in reduction of symptom severity between the two approaches, with a reoperation rate of $6 \% .^{2}$ In a recent meta-analysis, Vasiliadis reported an overall complication rate of $3.84 \%$ over 7227 patientyears. ${ }^{25}$ Additionally, a meta-analysis by Zuo et al. utilized 


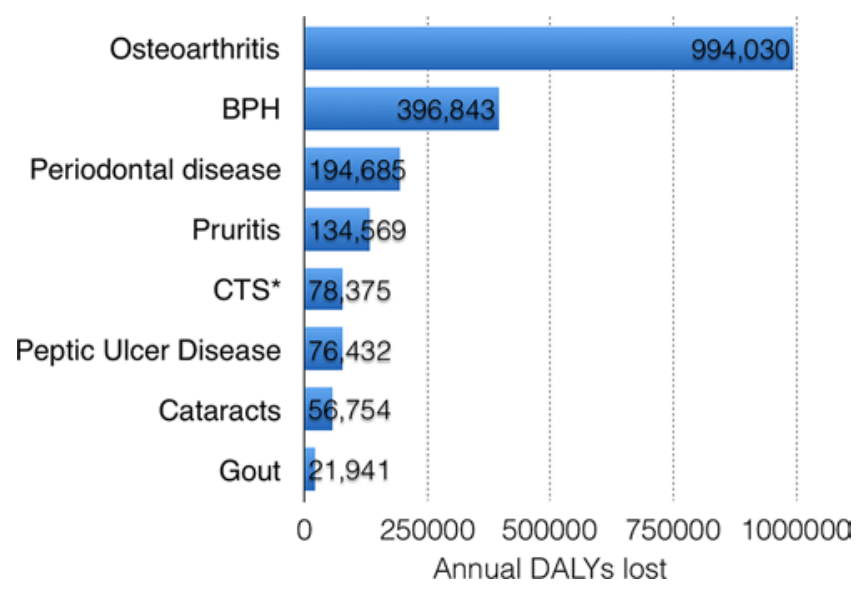

FIG. 3. Horizontal bar chart demonstrating annual disease burden in DALYs/year of various medical conditions. BPH = benign prostatic hypertrophy.

four studies to estimate patient subjective satisfaction at $87.96 \%$ and $87.97 \%$ for ECTR and OCTR, respectively. ${ }^{27}$

Overall, both OCTR and ECTR provide successful surgical management of CTS. In 1998, Katz et al. suggested a surgical success rate of $>70 \%$, which was later supported by Bland in 2007 who reported a rate of $75 \% .^{4,12}$ Subsequent studies suggested that CTS surgery appears to be corrective or obtain adequate relief of symptoms in 70\%$90 \% .^{15,16,18}$ The authors believe that a surgical success rate of $70 \%-80 \%$ can be used to conservatively estimate the economic benefit of OCTR and ECTR. Therefore, the most conservative estimate of CTS surgery (70\%) would provide between $\$ 3.73$ and $\$ 8.34$ billion in net economic benefit. This amounts to a benefit-cost ratio of $2.7: 1$ to 4.9:1.

Our study found a large economic burden of CTS within the Medicare population. CTS disease burden is greater than the annual burden of peptic ulcer disease, but less than conditions such as periodontal disease or benign prostatic hypertrophy (Fig. 3). ${ }^{11}$

OCTR and ECTR have provided a remarkable amount of benefit within the Medicare patient population. In the future, cost of treatment will likely be a main driver to utilization. This study indicated that surgical management is providing a large amount of benefit with a very low cost/ DALYs. Cost/DALYs was optimized when ECTR was utilized, however, this is functioning under the assumption that both procedures are providing equivalent improvement in the health state. Future studies may provide further insight into the optimal treatment modality and will be able to delineate which approach is most cost-effective.

\section{Limitations}

This study is not without limitations. The PearlDiver database is reliant on accurate CPT or ICD coding, which creates the potential for a reporting bias. In addition, we investigated charges and reimbursements rather than direct costs because this was not available within the PearlDiver database. Finally, patient comorbidities were not stratified within the scope of this study.
The limitations of DALYs are well described in previous literature. ${ }^{1}$ The DALY was not intentioned to quantify reduction of the burden of disease. ${ }^{26}$ The authors believe that this model is a reasonable assessment of the economic benefit in treating CTS, as surgical management has an established efficacy. The disability weight was established in surveying 30,660 people, which is likely representative of the patient perception of healthy years of life loss due to the condition. This analysis is limited in the fact that a large number of Medicare patients incorporated were subject to surgical management by two different modalities. Each modality may be performed by a litany of different techniques and/or systems, by many different surgeons. We attempted to account for this variability by calculating the economic benefit across increasing proportions of surgical success.

\section{Conclusions}

This study indicates there is a great amount of economic benefit provided by performing OCTR and ECTR for CTS within the Medicare patient population. The total annual DALYs for CTS are comparable to that of peptic ulcer disease, pruritus, and cataracts. Each patient treated provides $\$ 7498$ to $\$ 22,132$ in benefit for OCTR and $\$ 8178$ to $\$ 23,186$ in benefit for ECTR depending on the surgical success rate. The cost-benefit ratio, therefore, is 2.7:1 with most conservative estimates, and potentially as high as 6.9:1.

\section{References}

1. Anand S, Hanson K: Disability-adjusted life years: a critical review. J Health Econ 16:685-702, 1997

2. Atroshi I, Hofer M, Larsson GU, Ranstam J: Extended follow-up of a randomized clinical trial of open vs endoscopic release surgery for carpal tunnel syndrome. JAMA 314:1399-1401, 2015

3. Beck JD, Deegan JH, Rhoades D, Klena JC: Results of endoscopic carpal tunnel release relative to surgeon experience with the Agee technique. J Hand Surg Am 36:61-64, 2011

4. Bland JD: Treatment of carpal tunnel syndrome. Muscle Nerve 36:167-171, 2007

5. Devleesschauwer B, Havelaar AH, Maertens de Noordhout C, Haagsma JA, Praet N, Dorny P, et al: Calculating disability-adjusted life years to quantify burden of disease. Int $\mathbf{J}$ Public Health 59:565-569, 2014

6. Fajardo M, Kim SH, Szabo RM: Incidence of carpal tunnel release: trends and implications within the United States ambulatory care setting. J Hand Surg Am 37:1599-1605, 2012

7. Haagsma JA, Maertens de Noordhout C, Polinder S, Vos T, Havelaar AH, Cassini A, et al: Assessing disability weights based on the responses of 30,660 people from four European countries. Popul Health Metr 13:10, 2015

8. Hansen TB, Majeed HG: Endoscopic carpal tunnel release. Hand Clin 30:47-53, 2014

9. Ibrahim I, Khan WS, Goddard N, Smitham P: Carpal tunnel syndrome: a review of the recent literature. Open Orthop J 6:69-76, 2012

10. Kang HJ, Koh IH, Lee TJ, Choi YR: Endoscopic carpal tunnel release is preferred over mini-open despite similar outcome: a randomized trial. Clin Orthop Relat Res 471:15481554,2013

11. Kassebaum N, Arora M, Barber RM, Bhutta ZA, Brown J, Carter A, et al: Global, regional, and national disabilityadjusted life-years (DALYs) for 315 diseases and injuries and 
healthy life expectancy (HALE), 1990-2015: a systematic analysis for the Global Burden of Disease Study 2015. Lancet 388:1603-1658, 2016

12. Katz JN, Keller RB, Simmons BP, Rogers WD, Bessette L, Fossel AH, et al: Maine Carpal Tunnel Study: outcomes of operative and nonoperative therapy for carpal tunnel syndrome in a community-based cohort. J Hand Surg Am 23:697-710, 1998

13. Law TY, Rosas S, Hubbard ZS, Chieng LO, Chim HW: Trends in open and endoscopic carpal tunnel release utilization in the Medicare patient population. J Surg Res 214:913,2017

14. Lopez AD, Mathers CD, Ezzati M, Jamison DT, Murray CJL (eds): Global Burden of Disease and Risk Factors. New York: Oxford University Press, 2006

15. Louie D, Earp B, Blazar P: Long-term outcomes of carpal tunnel release: a critical review of the literature. Hand (N Y) 7:242-246, 2012

16. Louie DL, Earp BE, Collins JE, Losina E, Katz JN, Black EM, et al: Outcomes of open carpal tunnel release at a minimum of ten years. J Bone Joint Surg Am 95:1067-1073, 2013

17. Mintalucci DJ, Leinberry CF Jr: Open versus endoscopic carpal tunnel release. Orthop Clin North Am 43:431-437, 2012

18. Mosier BA, Hughes TB: Recurrent carpal tunnel syndrome. Hand Clin 29:427-434, 2013

19. Murray CJ: Quantifying the burden of disease: the technical basis for disability-adjusted life years. Bull World Health Organ 72:429-445, 1994

20. Murray CJ, Acharya AK: Understanding DALYs (disabilityadjusted life years). J Health Econ 16:703-730, 1997

21. Phalen GS: The carpal-tunnel syndrome. Seventeen years' experience in diagnosis and treatment of six hundred fiftyfour hands. J Bone Joint Surg Am 48:211-228, 1966

22. Smetana BS, Zhou X, Hurwitz S, Kamath GV, Patterson JM: Effects of hand fellowship training on rates of endoscopic and open carpal tunnel release. J Hand Surg Am 41:e53e58, 2016

23. Thoma A, Veltri K, Haines T, Duku E: A meta-analysis of randomized controlled trials comparing endoscopic and open carpal tunnel decompression. Plast Reconstr Surg 114:1137-1146, 2004
24. Trumble TE, Diao E, Abrams RA, Gilbert-Anderson MM: Single-portal endoscopic carpal tunnel release compared with open release: a prospective, randomized trial. J Bone Joint Surg Am 84-A:1107-1115, 2002

25. Vasiliadis HS, Nikolakopoulou A, Shrier I, Lunn MP, Brassington R, Scholten RJ, et al: Endoscopic and open release similarly safe for the treatment of carpal tunnel syndrome. A systematic review and meta-analysis. PLoS One 10:e0143683, 2015

26. Warf BC, Alkire BC, Bhai S, Hughes C, Schiff SJ, Vincent JR, et al: Costs and benefits of neurosurgical intervention for infant hydrocephalus in sub-Saharan Africa. J Neurosurg Pediatr 8:509-521, 2011

27. Zuo D, Zhou Z, Wang H, Liao Y, Zheng L, Hua Y, et al: Endoscopic versus open carpal tunnel release for idiopathic carpal tunnel syndrome: a meta-analysis of randomized controlled trials. J Orthop Surg Res 10:12, 2015

\section{Disclosures}

The authors report no conflict of interest concerning the materials or methods used in this study or the findings specified in this paper.

\section{Author Contributions}

Conception and design: Hubbard, Chim. Acquisition of data: Hubbard, Law, Rosas, Chim. Analysis and interpretation of data: Hubbard, Law, Jernigan. Drafting the article: Hubbard, Law, Jernigan, Chim. Critically revising the article: all authors. Reviewed submitted version of manuscript: all authors. Approved the final version of the manuscript on behalf of all authors: Hubbard. Statistical analysis: Hubbard. Administrative/technical/ material support: Hubbard, Jernigan, Chim. Study supervision: Hubbard, Jernigan, Chim.

\section{Correspondence}

Zachary St. Hubbard: University of Miami Miller School of Medicine, Fort Lauderdale, FL. z.hubbard@med.miami.edu. 